REVIEW

\title{
Autonomic changes in patients with heart failure and in post-myocardial infarction patients
}

\section{P Frenneaux}

Heart 2004;90:1248-1255. doi: 10.1136/hrt.2003.026146

A range of techniques for the assessment of autonomic tone are described. Impaired baroreflex control of heart period, measured in terms of heart rate variability or baroreflex sensitivity, is independently associated with adverse prognosis in patients with heart failure and following acute myocardial infarction. These techniques have not yet entered routine clinical practice.

input to the brainstem is to promote increased sympathetic outflow and to reduce vagal outflow from the brainstem. There is also altered central gain of the baroreflex, promoting sympathoexcitation. Reduced brainstem nitric oxide and increased angiotensin II appear to play an important role in this change. Finally, there is evidence of altered function of vagal ganglia.

The disturbances of afferent input from arterial baroreceptors and cardiopulmonary mechanoreceptors and of central gain are measurable as a reduced response of heart period (RR interval) to a change in blood pressure (reduced cardiac baroreflex sensitivity (BRS)). A series of elegant studies in an experimental canine postMI model have suggested that low BRS is a very powerful determinant of susceptibility to ventricular fibrillation during episodes of myocardial ischaemia, and that interventions which increase BRS reduce the susceptibility to ventricular fibrillation. ${ }^{2}$ An extensive body of literature now exists in humans suggesting that impaired cardiac baroreflex control and sympathoexcitation are associated with adverse outcome in patients following MI and in those with heart failure. In this review the techniques which may be employed to assess autonomic function in this setting will be described and the evidence base linking these measurements to outcome will then be summarised.

\section{CLINICAL ASSESSMENT OF SYMPATHOVAGAL BALANCE}

Measurements of sympathetic outflow to the periphery are useful in research studies (for example, muscle or renal sympathetic nerve activity, measurement of noradrenaline spillover, and clearance using radioactive tracer techniques) but are impractical for routine clinical use. The techniques which have been used clinically focus on the autonomic modulation of heart period. central (brainstem) gain, and altered efferent effector mechanisms.

Evidence from experimental heart failure models suggest that there is reduced input from cardiopulmonary receptors with vagal afferents and from arterial baroreceptors. The tonic activity of these receptors in the normal state has a restraining effect on sympathetic outflow from the brainstem; reduced afferent activity therefore promotes sympathoexcitation. Conversely, there is increased activity in those afferents which promote sympathetic outflow from the brainstem, including cardiac sympathetic afferent fibres, and afferents from skeletal muscle metaboreceptors and peripheral and central chemoreceptors. The net effect of this altered afferent

Abbreviations: $A C E$, angiotensin converting enzyme; AICD, automatic implantable cardioverter-defibrillator; ATRAMI, autonomic tone and reflexes after myocardial infarction; ASDNN, average of the standard deviation of $\mathrm{NN}$ intervals; BRS, baroreflex sensitivity; $\mathrm{Cl}$, confidence interval; HRV, heart rate variability; HF, high frequency; LVEF, left ventricular ejection fraction; LF, low frequency $\mathrm{MI}$, myocardial infarction; RR, relative risk; rMSSD, root mean square successive difference; SDANN, standard deviation of average NN intervals; SDNN, standard deviation of NN intervals; TINN, triangular interpolation of NN intervals; TO, turbulence onset; TS, turbulence slope; ULF, ultra low frequency; VF, ventricular fibrillation; $\mathrm{VLF}$, very low frequency; VPC, ventricular premature contraction 
Four forms of assessment have been shown to be useful in clinical practice for risk factor stratification (see box for summary):

- Assessment of heart rate (or more correctly heart period) variability

- Assessment of cardiac BRS

- Assessment of heart rate responses during exercise and during recovery.

- Heart rate turbulence

\section{Heart rate variability (HRV)}

The variability of RR intervals gives important information about the autonomic control of heart period. This variability may be assessed by time domain, frequency domain, or by non-linear techniques.

Time domain techniques

Commercially available systems are able to distinguish between $\mathrm{R}$ waves from sinus beats and those from non-sinus beats. Only sinus beats are included in the analysis. The time

\section{Clinical assessment of autonomic control of} heart period: summary

Heart rate variability

- Time domain indices of HRV

Statistical measures

- SDNN: standard deviation of NN intervals

- SDANN: standard deviation of average NN intervals

- ASDNN: average of the standard deviation of NN intervals

- NN50: number of adjacent NN intervals which differ by at least $50 \mathrm{~ms}$ during a 24 hour recording.

- pNN50: percentage of adjacent NN intervals in a 24 hour recording which differ by at least $50 \mathrm{~ms}$

- rMSSD: root mean square of successive differences.

Geometric time domain measures

- HRV triangular index

- TINN: trinangular interplation of NN intervals

- Lorenz (Poincare) plots

- Frequency domain techniques

- Non-linear dynamic methods (application of chaos theory)

\section{Cardiac baroreflex sensitivity}

- Phenylephrine bolus technique

- Spontaneous sequence technique

- Cross spectral technique

Heart rate response during exercise and during recovery

- Chronotropic incompetence

- Chronotropic response index

- Heart rate recovery response

Heart rate turbulence

- Turbulence onset

- Turbulence slope interval between consecutive normal (N) (sinus rhythm) R waves is measured by the software and is known as the NN interval. The technique can be applied to long ( 24 hour) or short recordings. The NN intervals are then used to calculate time domain indices of HRV. These can be divided into statistical and geometric time domain measurements.

\section{Statistical time domain measurement}

Standard statistical measurements ${ }^{3}$ can be applied to the NN interval dataset to express the variability. The standard deviation of NN intervals ( SDNN) is of course related to the length of recording, the longer the recording period the greater the SDNN. The SDNN is usually applied to 24 hour recordings, and if the recording period is shorter this must be clearly stated. SDNN is a frequently used measure.

The standard deviation of average NN intervals (SDANN) is useful for assessing variations in heart period with cycle lengths longer than five minutes. NN intervals are grouped into five minute segments (288 segments in each 24 hour period) and the mean $\mathrm{NN}$ interval for each five minute segment is calculated. The SDANN is the standard deviation of these 288 means.

The average of the standard deviation of $\mathrm{NN}$ intervals (ASDNN) is useful for assessing variations in heart period with cycle lengths shorter than five minutes. It is the average of the standard deviations of the NN intervals for the 288 five minute segments in a 24 hour period.

The number of adjacent NN intervals which differ by at least $50 \mathrm{~ms}$ during a 24 hour recording is known as the NN50. The percentage of adjacent NN intervals in a 24 hour recording which differ by at least $50 \mathrm{~ms}$ is the pNN50; this appears to be a particularly good measure of cardiac vagal tone. Each NN interval during a 24 hour recording is squared, the mean of these squared NN intervals is then derived, and the square root of this mean is calculated and is called the root mean square successive difference (rMSSD).

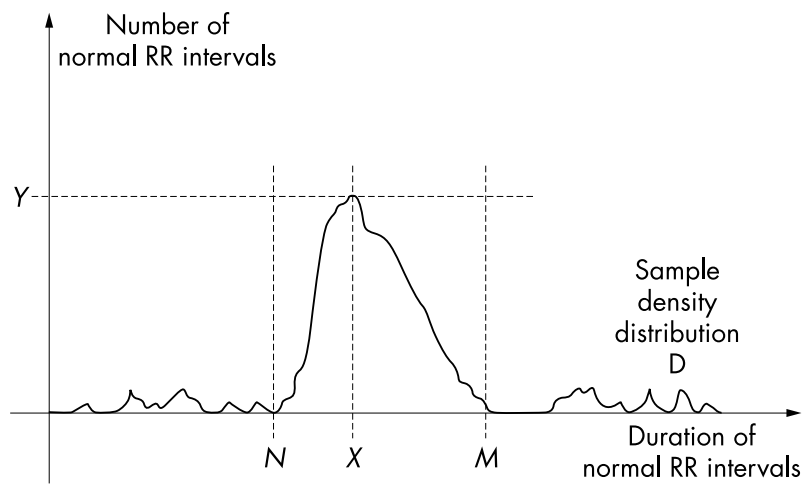

Figure 1 Measurement of geometric time domain indices of heart rate variability (HRV). To perform geometric measures on the $\mathrm{NN}$ interval histogram, the sample density distribution D is constructed, which assigns the number of equally long $N N$ intervals to each value of their lengths. The most frequent NN interval length $X$ is established-that is, $Y=D(X)$ is the maximum of the sample density distribution $D$. The HRV triangular index is the value obtained by dividing the area integral of $D$ by the maximum $Y$. When the distribution $D$ with a discrete scale is constructed on the horizontal axis, the value is obtained according to the formula HRV index $=$ (total number of all $N N$ intervals) $/ Y$. For the computation of the triangular interpolation of NN intervals (TINN) measure, the values $N$ and $M$ are established on the time axis and a multi-linear function $q$ constructed such that $q(t)=0$ for $t N$ and $t M$ and $q(X)=Y$, and such that the integral $0+D(t)-q(t)) 2 d t$ is the minimum among all selections of all values $N$ and $M$. The TINN measure is expressed in milliseconds and given by the formula TINN $=M-N$. Reproduced with permission from Task Force of the European Society of Cardiology and the North American Society of Pacing and Electrophysiology. Circulation 1996;93:1043-65. 


\section{Geometric time domain measurement}

A frequency histogram of NN intervals throughout a 24 hour period is constructed as shown in fig 1. At a standard sampling rate of $128 \mathrm{~ms}$ the width of each bin in the histogram is 7.8125 ms. $^{4}$

HRV triangular index is calculated by dividing the total number of $\mathrm{NN}$ intervals in the 24 hours by the modal frequency in the frequency histogram.

To calculate TINN a triangle is constructed which best fits the frequency histogram (using a least squares technique); TINN is the width of the base of this triangle.

A Lorenz (or Poincare) plot is illustrated in fig 2. A plot of the current $\mathrm{NN}$ interval ( $\mathrm{x}$ axis) versus the subsequent $\mathrm{NN}$ interval (y axis) is constructed. In healthy states, the disparity between current and next NN interval becomes progressively greater at higher $\mathrm{NN}$ intervals, whereas in disease states (for example heart failure) this is not so.

\section{Frequency domain techniques}

Although these techniques can be used for the assessment of both short and long term recordings, frequency domain techniques $^{3}$ are particularly useful for the assessment of relatively short recordings (several minutes). These short term recordings provide valuable information about autonomic control of heart period. As with time domain techniques the RR intervals are measured. Dummy intervals are interpolated for non-sinus beats (based on the previous and following beats). Parametric (autoregression) or nonparametric (fast Fourier transformation) techniques may be applied to the data to examine the oscillatory behaviour of heart period. Both techniques produce a power spectral density of the RR intervals. An example is shown in fig 3. On the $\mathrm{x}$ axis is the frequency of RR variability $(\mathrm{Hz})$ and on the $y$ axis the power, which is the square of the amplitude (expressed in $\mathrm{ms}^{2}$ ). When applied to short (approximately five minute) recordings, frequencies between $0.033-0.4 \mathrm{~Hz}$ can be evaluated. As shown in fig 3, three prominent peaks are apparent in a healthy subject, in the supine position; a high frequency (HF) peak between $0.15-0.4 \mathrm{~Hz}$, a larger low frequency (LF) peak between $0.04-0.15 \mathrm{~Hz}$, and an even larger very low frequency (VLF) peak between 0.0033$0.04 \mathrm{~Hz}$. The power of these spectra may be expressed either in absolute units or as a proportion of total power (normalised units).

There is little controversy regarding the origin of the HF peak. It principally represents vagal modulation of heart period in response to ventilation (respiratory sinus arrhythmia). However, even in denervated hearts some HF variability

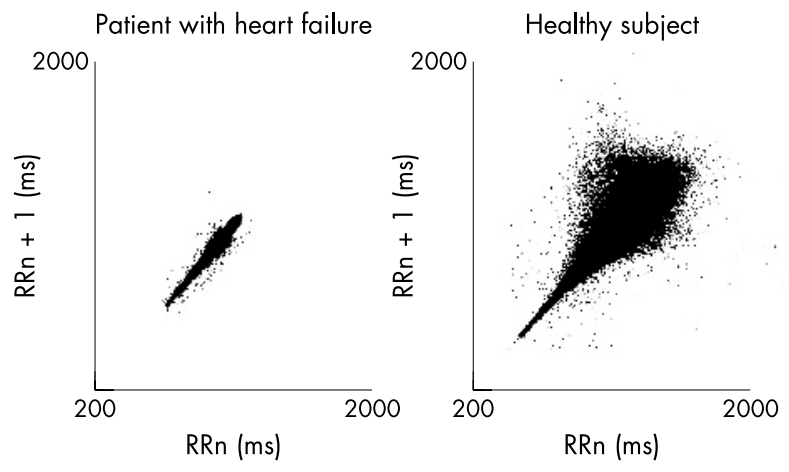

Figure 2 Lorenz (Poincare) plot. The current NN interval (x axis) is plotted against the previous NN interval (y axis). In healthy subjects there is a notable disparity between the two NN intervals; at longer NN intervals lie low heart rates. This is not so in patients with heart failure. Reproduced with permission from Woo MA, et al. J Am Coll Cardiol 1994;23:565-9. may be apparent, especially on exercise, and this is probably caused by the effect of changes in atrial transmural pressure during the respiratory cycle on sinus node automaticity. ${ }^{5}$

The origin of LF variability is more contentious. In uncomplicated MI short term recordings reveal a predominant LF and a smaller HF component, with a LF/HF ratio $>2$. Originally it was asserted that the magnitude of the LF peak or the LF/HF ratio reflected sympathetic modulation of heart period, but this is clearly incorrect because absolute LF power is reduced in heart failure and during exercise, both situations being associated with elevated sympathetic tone. Indeed, in patients with severe heart failure total power is diminished and there is typically a small or absent LF component and a relatively predominant HF component, with $>60-70 \%$ of the power in the VLF range. ${ }^{6}$ LF power probably reflects in large part baroreflex modulation of heart period in response to spontaneous changes in blood pressure by both vagal and sympathetic efferent mechanisms. ${ }^{78}$ The precise origin of the VLF peak is unknown.

During such short term recordings, acquisition conditions must be standardised in order to optimise the quality of the data and to aid in its interpretation. Recordings should be undertaken following 30 minutes of supine rest. Respiratory frequency should be recorded or alternatively, during acquisition, controlled breathing at 12 to 15 breaths per minute should be undertaken (with the aid of a metronome). Relatively high data acquisition sampling frequencies are required (at least $250 \mathrm{~Hz}$ and typically $500 \mathrm{~Hz}$ or higher).

As noted above, frequency domain techniques may also be applied to longer recordings. During 24 hour recordings an additional spectral peak is evident (below $0.0033 \mathrm{~Hz}$ ) termed the ultra low frequency peak (ULF). The origin of this spectral peak is controversial. There is some evidence that it is influenced by thermoregulatory mechanisms and by the renin-angiotensin system, but physical activity also notably influences power in this spectral frequency range. ${ }^{9}$ During 24 hour recordings LF and HF components represent $<10 \%$ of total spectral energy.

In general, HF power correlates closely with both pNN50 and rMSSD, these parameters each providing information about the vagal control of heart period in response to respiration. ULF power generally correlates with SDNN and SDANN, and LF power generally correlates with ASDNN.

\section{Non-linear dynamics}

Heart period does not show completely regular periodicity explicable solely on the basis of a linear approach. Non-linear approaches (based on chaos theory) have been applied to the study of heart period and have shown that apparently erratic behaviour may actually be generated by a deterministic system with non-linear structure. These studies have shown that fluctuations in RR intervals may be related to variations that occurred hundreds of beats earlier and that these are dependent on the functional integrity of baroreflex mechanisms. Detailed description of these techniques is beyond the scope of this review, but recent studies have suggested that such an approach may accurately identify post-MI patients at high risk of mortality-indeed they may be more predictive than traditional time and frequency domain methods. ${ }^{10}$

\section{Cardiac baroreflex sensitivity}

BRS provides a measure of the gain of the reflex arc which modulates heart period in response to changes in blood pressure. In fact, the relation between blood pressure and heart period is sigmoid, with a steep central component which is approximately linear, and upper and lower plateaus. In research studies the full sigmoid relation is sometimes delineated, ${ }^{11}$ but in clinical studies this is not the case. Unless the full range is explored then a reduction in BRS may reflect either a reduction in gain of the central (steep) component of 


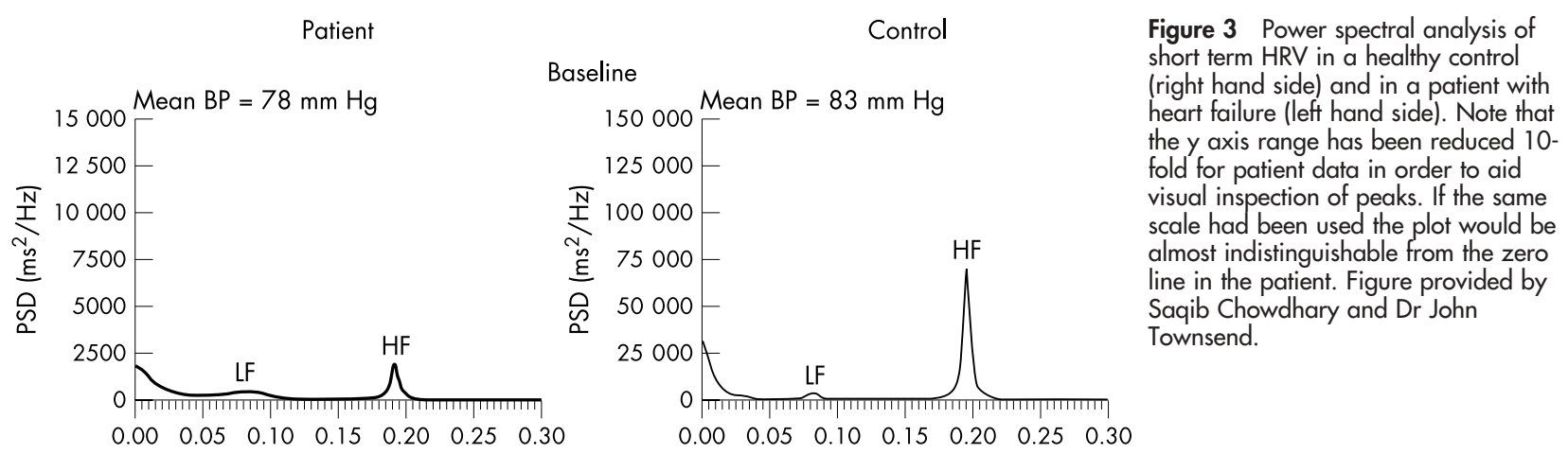

the relation or a change in the operating position towards a flatter part of the sigmoid relation. In general, impaired BRS is associated with reduced cardiac vagal control and with increased sympathetic efferent activity.

As with assessment of HRV, standardisation of conditions is important. Studies should be performed after 30 minutes of supine rest in a quiet environment.

\section{Phenylephrine bolus technique}

In the original studies pioneered by the Oxford group, systolic blood pressure was increased by $20-30 \mathrm{~mm} \mathrm{Hg}$ using an intravenous bolus of phenylephrine (approximately 50$150 \mu \mathrm{g}$ ). Arterial blood pressure was measured using an intra-arterial cannula. RR interval is plotted against the preceding systolic blood pressure for the ramp phase in which blood pressure is increasing, and a linear regression line is constructed. Only those ramps in which the $r^{2}$ of the regression lines $>0.85$ are included. ${ }^{12}$ Generally BRS is calculated from the mean of three such acceptable regression slopes obtained by bolus phenylephrine injections approximately five minutes apart, and is expressed in $\mathrm{ms} / \mathrm{mm} \mathrm{Hg}$. A typical study is shown in fig 4. More recently intra-arterial pressure recording has been replaced by non-invasive beat by beat blood pressure recordings using devices such as the Finapres and Portapres, which have been shown to provide reliable measurements for assessing BRS. Some studies have examined the gain of the reflex in response to a fall in blood pressure-for example, by the nitric oxide donor sodium nitroprusside. However, recent data suggests that nitric oxide donors may themselves directly modify the gain of the reflex (via effects on arterial distensibility, baroreflex nerve firing, central gain in the brainstem, and sinus node automaticity). ${ }^{13}$

The phenylephrine bolus technique remains the gold standard technique, having been used in the major studies linking BRS to prognosis in post-MI and heart failure patients.

\section{Spontaneous sequence analysis}

This technique examines the gain of the cardiac baroreflex during spontaneous variations in blood pressure rather than in response to drug induced changes in blood pressure. Noninvasive beat by beat blood pressure (Finapres or Portapres) and ECG are recorded. Commercially available software programs are able to identify spontaneous sequences in which blood pressure and RR interval concurrently increase (up sequences) or decrease (down sequences) for three or more consecutive beats. Linear regression plots of systolic blood pressure against RR interval are then constructed. In most cases systolic blood pressure is plotted against the same RR interval (lag 0), but at higher heart rates it may be more appropriate to plot systolic blood pressure against the subsequent RR interval (lag 1). BRS is calculated from the mean of at least three slopes with $r^{2}>0.85 .{ }^{14}$ It has been validated against the phenylephrine technique and shown to be more reproducible with fewer test failures, although the absolute values for BRS obtained with the sequence technique are higher than with the phenylephrine technique. ${ }^{15} \mathrm{~A}$ disadvantage of both the phenylephrine and spontaneous sequence techniques is that in patients with very low BRS it may be very difficult to identify slopes during which systolic blood pressure and RR interval are changing concurrently. In these circumstances it may be impossible to measure BRS.

\section{Cross spectral techniques (frequency domain)}

Non-invasive beat by beat blood pressure, ECG and respiration are recorded for 5-8 minutes during controlled breathing (typically 20 breaths/min). Power spectral analysis of 256 beat segments of both blood pressure and RR interval are performed using autoregression analysis as described above. Coherence analysis is then used to assess the correlation
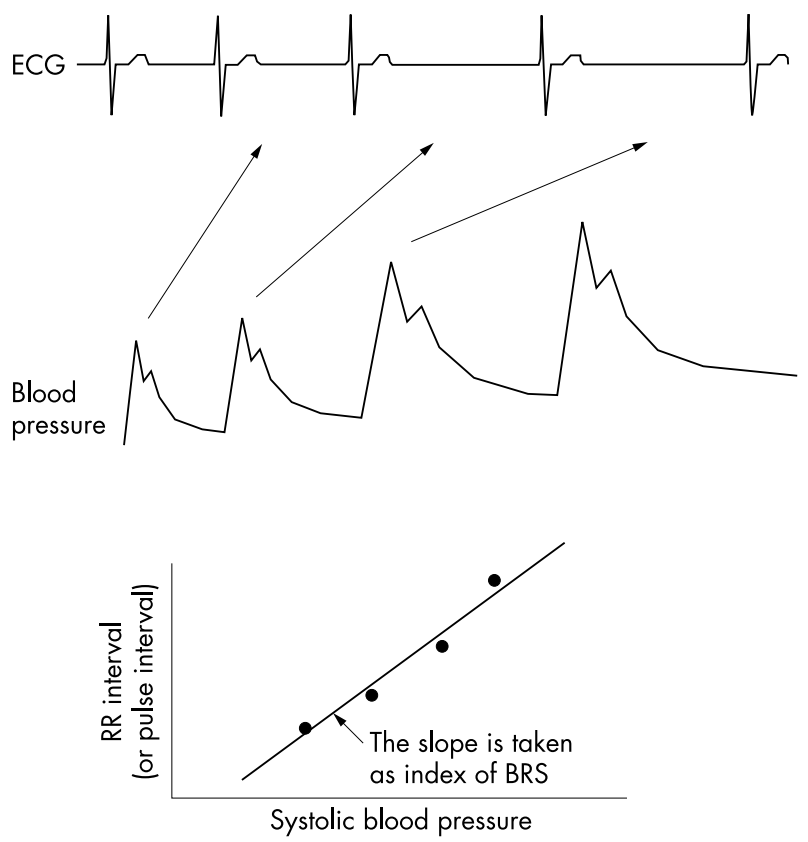

Figure 4 Phenylephrine bolus technique for measuring baroreflex sensitivity (BRS). After a bolus injection of phenylephrine there is a progressive increase in blood pressure and a reflex slowing of heart rate. BRS is calculated as the slope of the relation between systolic blood pressure and the subsequent $R R$ interval during this ramp increase in blood pressure. Reproduced with permission from Persson PB, et al. J Hypertens 2001;19:1699-705. 

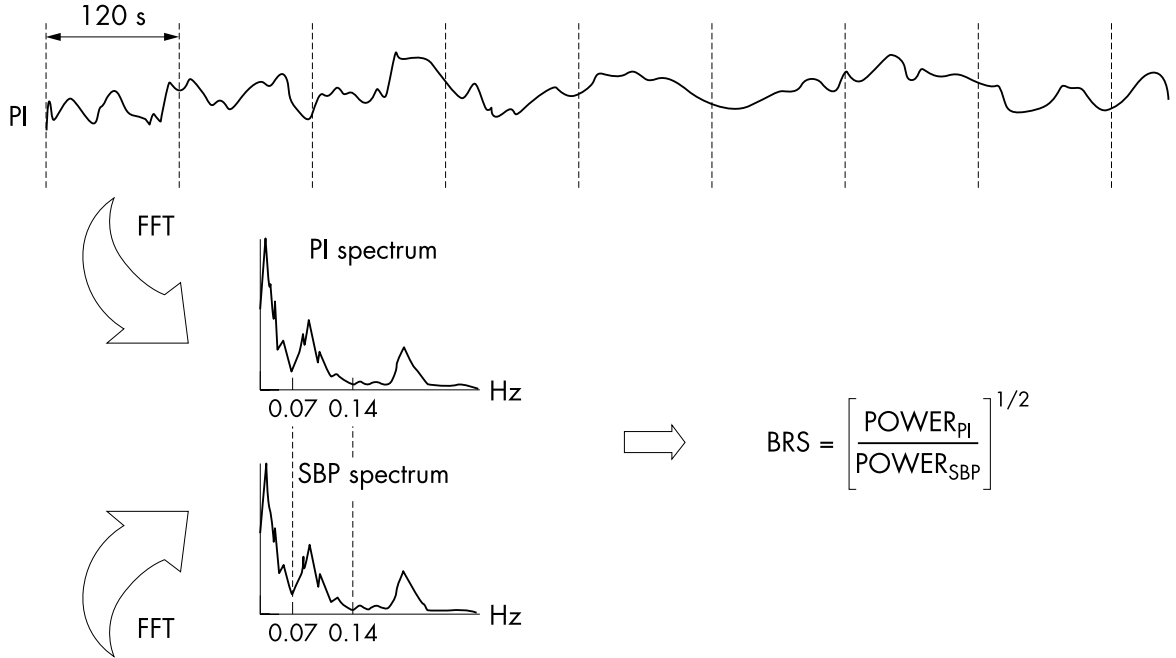

$$
B R S=\left[\frac{P_{\text {OWER }}}{\text { POWER }_{S B P}}\right]^{1 / 2}
$$

Figure 5 Cross spectral analysis of BRS. Power spectral analysis of blood pressure and heart period. Cross spectral analysis is used to measure BRS as shown. Reproduced with permission from Parati $G$, et al. J Hypertens 2000;18:7-19.

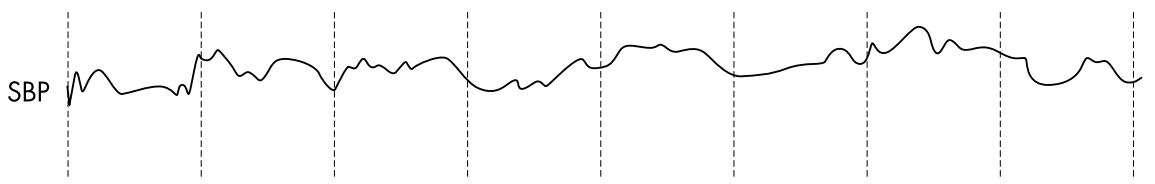

among oscillations in RR interval and systolic blood pressure. The squared coherence function evaluates the phase stability of pairs of oscillations with the same frequency, present in two signals (in this case systolic blood pressure and RR interval). It can range from 0 (no correlation) to 1 (complete coherence). High squared coherence values between variations in systolic blood pressure and RR interval in the LF band reflect an intact baroreflex control of heart period and it is then possible to calculate BRS using the $\alpha$ index, which is the square root of the ratio of RR interval power to systolic blood pressure power in the LF and/or HF bands (fig 5). ${ }^{16-18}$ In one recent study BRS estimated from the spontaneous sequence and $\alpha$ index techniques was shown to be similar, but there were fewer test failures with the latter technique. ${ }^{15}$

Nevertheless, in patients with low BRS (as in heart failure and post-MI patients), test failure is relatively common because of low squared coherence function. Recently a modification of the cross spectral technique was described called wide band averaging, which has the potential to largely overcome this problem. In this technique the average systolic blood pressure/RR interval transfer function is assessed over a relatively wide frequency band region $(0.04-0.15 \mathrm{~Hz})$ without considering the coherence between the two signals. The BRS measurements obtained using this technique were shown to correlate reasonably well with those obtained using the phenylephrine bolus technique. ${ }^{19}$

\section{Assessment of heart rate responses during exercise and during recovery}

A blunted heart rate response to exercise appears to reflect heightened basal sympathetic tone, and may in part be caused by $\beta$ receptor desensitisation. ${ }^{20}$ Chronotropic incompetence is defined as a failure to achieve $85 \%$ of age and sex predicted peak heart rate during maximal exercise. ${ }^{21}$ Although chronotropic incompetence has been shown to be related to prognosis in population studies, ${ }^{21}$ it is less reliable when applied on an individual basis because of a wide standard deviation. The chronotropic response index is the ratio of heart rate reserve used and metabolic reserve used during exercise. ${ }^{21}$ The two parameters are linearly related, and in healthy subjects the ratio is approximately 1 . An abnormal chronotropic response index is defined as a ratio
$<0.8$. Unlike peak heart rate it is independent of resting heart rate and of level of fitness.

The reduction in heart rate during the first 30-60 seconds following exercise appears to be principally caused by recovery of parasympathetic tone but subsequently withdrawal of sympathetic tone appears to play an important role. ${ }^{22}$ Several population based studies have shown a relation between impaired heart rate recovery following exercise and increased cardiac mortality. For example, in a recent study of over 2000 male patients referred for evaluation of chest pain, a heart rate reduction of $<22 \mathrm{bpm}$ at two minutes postexercise was associated with a hazard ratio of 2.6 (95\% confidence interval (CI) 2.4 to 2.8) for total mortality during a mean 7 year follow up. ${ }^{23}$

\section{Heart rate turbulence}

Heart rate turbulence refers to the phenomenon whereby there is an initial acceleration followed by a deceleration of sinus rhythm following a ventricular premature contraction (VPC). This is because when a VPC appears early in the cardiac cycle there is a reduction in stroke volume which leads to an initial baroreflex mediated reduction in vagal outflow. Conversely, stroke volume is increased by the compensatory pause and this leads to a subsequent increase in vagal outflow. ${ }^{24}$ The parameter turbulence onset (TO) expresses the relative shortening of RR intervals immediately after the compensatory pause. Turbulence slope (TS) expresses the slope of the linear regression line between RR interval count and RR interval duration during the subsequent lengthening of RR intervals. ${ }^{25}$

\section{PROGNOSTIC SIGNIFICANCE OF AUTONOMIC FUNCTION TESTS IN PATIENTS FOLLOWING MI}

Several studies have demonstrated a relation between abnormalities of RR variability or of impaired BRS and adverse prognosis in patients following MI. These studies are consistent with data from a canine post-MI model in which BRS is a potent predictor of the susceptibility to ventricular fibrillation (VF) during myocardial ischaemia. Interestingly, although MI itself substantially reduces BRS, data from this model shows that pre-morbid BRS (that is, before the MI) is 
also an important predictor of the VF risk during the subsequent studies performed post-MI.

A large study published in 1987 (that is, performed in the pre-thrombolytic era) demonstrated that patients with a SDNN of $<50 \mathrm{~ms}$, measured at an average lldays post-MI, had a relative risk (RR) of mortality during 2-4 year follow up of 2.8 (95\% CI 2 to 3.8$).{ }^{26}$ In two other small studies published in 1988 and 1992, a low BRS predicted total cardiac mortality and life threatening arrhythmic events respectively..$^{27}$

However, the strongest evidence comes from the ATRAMI (autonomic tone and reflexes after myocardial infarction) study. A total of 1248 patients with a recent ( $<28$ days) MI underwent assessment of time domain indices of HRV and of BRS using the phenylephrine technique (with arterial cannulation). The patients were a relatively low risk group (mean left ventricular ejection fraction (LVEF) 49\%) and consistent with this, during the mean follow up of 21 months there were only 44 cardiac deaths and five non-fatal cardiac arrests. BRS $<? 3 \mathrm{~ms} / \mathrm{mm} \mathrm{Hg}$ and SDNN $<70 \mathrm{~ms}$ were both potent univariate predictors of cardiac mortality at one and two years $(\mathrm{p}<0.0001$ for each at two years $)$. In a multivariate model which included other prognostic variables (LVEF and VPC $>10 /$ hour) both measures of autonomic function remained significant predictors of cardiac mortality (RR 2.8, 95\% CI 1.24 to 6.16 for BRS $<3 \mathrm{~ms} / \mathrm{mm} \mathrm{Hg}$, and RR $3.2,95 \%$ CI 1.42 to 7.36 for SDNN $<70 \mathrm{~ms})$. In younger patients $(<65$ years $)$ the combination of LVEF $<35 \%$ and either low BRS or low SDNN was associated with a very high mortality rate (RR $11.5,95 \%$ CI 4.86 to 27.1 , and RR 5.9, 95\% CI 1.41 to 24.7 , respectively). Elderly patients were underrepresented in this study and this, together with the lower BRS in this age group, restricted the power of the study to examine the predictive value of BRS in the $>65$ age group. ${ }^{28}$ In a subsequent analysis of the ATRAMI dataset, the group showed that the combination of non-sustained ventricular tachycardia, depressed BRS, and reduced SDNN was associated with an RR of 22 for cardiac mortality on multivariate analysis. $^{28}$

Frequency domain measures of HRV have also been shown to be associated with adverse prognosis. In one study frequency domain HRV was performed on two, five minute epochs (one during the day and one during the night) from a 24 hour ECG recording obtained at approximately two weeks post-MI. Reduced HRV values predicted higher total mortality during 31 months follow up (2-4 fold RR). ${ }^{29}$

Non-linear fractal parameters of abnormal HRV has also been shown to be predictive of increased mortality post-MI in a subgroup of 446 patients in the DIAMOND (Danish investigations of arrhythmia and mortality on dofetilide in survivors of myocardial infarction) study. The patients all had LVEF $<36 \%$. The short term fractal scaling exponent I was a stronger predictor of both total mortality, arrhythmic death, and non-arrhythmic death than conventional time and frequency domain HRV measures (RR 3.0, 95\% CI 2.5 to 4.2) during two year follow up. ${ }^{10}$

In an analysis of the ATRAMI dataset abnormal heart rate turbulence measures (TO and TS) predicted subsequent fatal and non-fatal cardiac arrest (RR for the combination of both 1.86, but the confidence interval overlapped zero), but importantly, in a multivariate model which included LVEF, a composite autonomic index (TO, TS, BRS, and SDNN) was associated with an RR of 8.67 (95\% CI 2.7 to 27.65) of fatal and non-fatal cardiac arrest. ${ }^{30}$

\section{Prognostic significance of disturbances of autonomic tone in patients with heart failure}

Mortara et al examined the prognostic significance of reduced BRS (phenylephrine technique) in patients with moderate to severe heart failure of ischaemic and non-ischaemic aetiology. BRS was obtainable in 282 of 303 eligible heart failure patients in sinus rhythm. Mean (SD) BRS was 3.9 (4.0) ms/ $\mathrm{mm} \mathrm{Hg}$ and was similar in patients with ischaemic and nonischaemic aetiologies. On multivariate modelling, BRS $<1.3 \mathrm{~ms} / \mathrm{mm} \mathrm{Hg}$ was significantly associated with total mortality after adjusting for non-invasive known risk factors (NYHA, LVEF, baseline RR interval and peak $\mathrm{VO}_{2}$ ), but not after adjusting for haemodynamics. Retrospective subgroup analysis suggested that BRS performed better as a predictor of mortality in patients with ischaemic aetiology. ${ }^{31}$

In the UK Heart Study, SDNN was found to be an independent predictor of all cause mortality in patients with heart failure (ischaemic and non-ischaemic) and evidence of left ventricular systolic dysfunction (LVEF $<45 \%$ ). Independent predictors of death because of progressive heart failure were low, SDNN less than the median for the group, $112 \mathrm{~ms}$, serum sodium and serum creatinine. Cardiothoracic ratio, left ventricular end diastolic diameter, the presence of non-sustained ventricular tachycardia, and serum potassium (but not SDNN) were found to be independent predictors of sudden cardiac death. ${ }^{32} 33$

In a recent study the prognostic significance of frequency domain indices of HRV in heart failure was assessed. During controlled breathing, eight minute recordings were obtained. In a derivation sample of 242 consecutive patients sudden cardiac death was independently predicted by a model that included LF power $\left(<13 \mathrm{~ms}^{2}\right)$ and left ventricular end diastolic diameter $>77 \mathrm{~mm}$. In a subsequent validation sample LF power remained a predictor of sudden cardiac death. In this sample, there were two independent predictors of sudden cardiac death during follow up on multivariate modelling-LF power $<11 \mathrm{~ms}^{2}$ and $>83$ ventricular premature beats per hour on Holter monitoring. ${ }^{34}$

\section{Effects of drug treatment on autonomic function in heart failure and post-MI}

Cardiac glycosides have been shown to enhance carotid baroreflex responses to changes in carotid sinus pressure in an experimental model of heart failure. ${ }^{35}$ In human heart failure cardiac glycosides have been shown to decrease muscle sympathetic nerve activity and plasma noradrenaline and to increase cardiac vagal control (as assessed by HRV). ${ }^{36}$ Despite these favourable autonomic effects, digoxin had no effect on mortality in the DIG (digitalis investigation group) trial..$^{38} \mathrm{~A}$ post-hoc analysis of the trial has reported reduced mortality in patients with low serum digoxin concentrations, but increased mortality in those with higher serum digoxin concentrations. ${ }^{39}$ It is possible that the effects of digoxin on mortality may represent a balance between favourable neurohumoral consequences versus adverse consequences of increased myocardial cytosolic calcium.

$\beta$ Blockers have not only a peripheral anti-adrenergic action, but also act centrally to increase cardiac vagal efferent activity and reduce sympathetic efferent activity. In a pig coronary occlusion model, intracerebral injection of propranolol reduced the incidence of VF. ${ }^{40}$ In humans $\beta$ blockers have been shown to increase HRV measures of cardiac vagal control and BRS. ${ }^{41-43}$ These effects would theoretically be expected to be more pronounced with lipophilic $\beta$ blockers, such as metoprolol, compared with hydrophilic agents such as atenolol.

Angiotensin II promotes sympathetic efferent outflow and reduces cardiac vagal outflow from the brainstem and enhances peripheral noradrenaline release. ${ }^{44}{ }^{45}$ As expected angiotensin converting enzyme (ACE) inhibitors therefore increase BRS and increase cardiac vagal control in both heart failure and post-MI..$^{46-48}$ In one recent study, candesartan had 
no significant effect on frequency or time domain indices of HRV, but increased BRS in patients with heart failure. ${ }^{49}$

Aldosterone infusion impairs BRS and promotes increased sympathetic efferent activity in animals and in man, in part via an action on baroreceptors nerve endings via release of an as yet unidentified endothelium dependent intermediate. ${ }^{50} 51$ As expected, spironolactone augments cardiac vagal control in heart failure, ${ }^{52}$ perhaps contributing to the beneficial effects seen on mortality in the RALES study.

Low doses of muscarinic antagonists such as scopolamine paradoxically increase cardiac vagal efferent activity ${ }^{53}$ and this is associated with increases in high frequency HRV and BRS. $^{54-57}$ Clinical trials are in progress, but in a post-MI animal model scopolamine did not reduce the risk of VF despite improving autonomic function..$^{55}$ Importantly, the autonomic tests described retain their prognostic significance in patients on contemporary medical therapies, including ACE inhibitors and $\beta$ blockers. ${ }^{32} 3358$

\section{Clinical role of tests of autonomic function in post-MI patients and in patients with heart failure}

None of the tests described have yet entered routine clinical use for risk factor stratification. Should these tests have a routine clinical role? It is certainly the case that they provide information about prognosis which is additional to that provided by conventional markers such as LVEF. Recent studies in patients with asymptomatic left ventricular dysfunction following MI and in patients with heart failure of both ischaemic and non-ischaemic aetiology have shown a survival benefit from the use of automatic implantable cardioverter-defibrillators (AICDs). ${ }^{4}$ The financial implications of applying this evidence base are, however, immense. The available evidence suggests that a population of patients at high risk of sudden cardiac death may be identified using HRV and/or BRS techniques. It may be cost effective to target AICD therapy to the subset of patients at higher risk of sudden cardiac death.

The recent MADIT 2 study undertaken in patients with prior MI and LVEF $<30 \%$ demonstrated that implanting an AICD significantly reduced mortality, but the resource implications are huge; 17 implants would save one life over two years. ${ }^{59}$ Based on an analysis of the ATRAMI database it may be possible to select a group of such patients at high risk of mortality (mainly arrhythmic) and a group at low risk of mortality. In the 157 patients with LVEF $<35 \%$ the overall two year mortality rate was only $11.4 \%$. However, the presence of either non-sustained ventricular tachycardia or impaired BRS increased the positive predictive accuracy (albeit to only $20 \%$ ) with an acceptable negative predictive accuracy $(95 \%)$. In summary, implementing such a strategy would mean that only $45 \%$ of the post-MI patients with LVEF $<45 \%$ or $<35 \%$ in the ATRAMI database would have undergone AICD implantation, and that the two year mortality in the group not implanted would have been $5 \% .^{34}$ There are three major caveats to such a strategy. Firstly, the absolute number of events was low in this sample; secondly, these data, acquired in a derivation sample, need to be confirmed in a separate validation sample; and thirdly, only $20 \%$ of patients were taking $\beta$ blockers at the time of assessment of autonomic function. $\beta$ Blockers increase BRS and in practice $\beta$ blocker use post-MI is now rather higher than this. The impact of this change on the prognostic utility of BRS is not clear. An additional issue is the use of the invasive phenylephrine technique with arterial cannulation. In the ATRAMI study a subgroup of 454 patients had Finapres recording of beat by beat blood pressure during phenylephrine injections as well as the standard intra-arterial recordings. There was good agreement between the invasive and non-invasive techniques of measuring BRS, and the

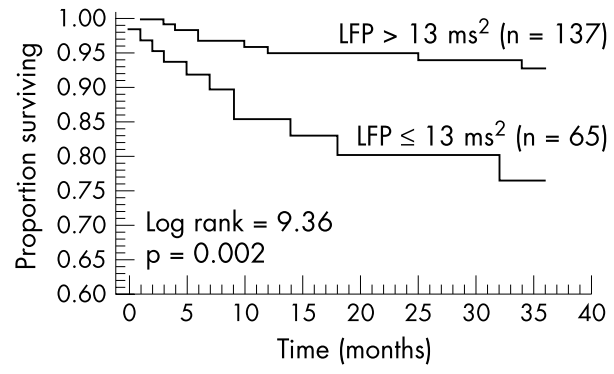

Figure 6 Prognostic significance of low frequency power (LFP) in heart failure. Reduced LFP $\left(\leqslant 13 \mathrm{~ms}^{2}\right)$ was associated with increased risk of sudden cardiac death in patients with heart failure. On multivariate modelling, reduced LFP and frequent ventricular premature beats ( $>83 /$ hour) were the two independent predictors of sudden cardiac death. Reproduced from La Rovere et al ${ }_{1}^{58}$ with permission.

non-invasive technique provided similar prognostic information. ${ }^{60}$ The spontaneous sequence or cross spectral techniques would be easier to use in clinical practice than the phenylephrine technique but produce generally higher measurements and their use for risk factor stratification post-MI has not been established in large studies.

The concept of using autonomic function tests to target treatment to high risk subsets of post-MI patients also receives some support from the results of a prospective substudy of EMIAT in patients with recent MI and LVEF $<41 \%$. SDNN (measured from 24 hour recordings) $<50 \mathrm{~ms}$ was associated with a more than twofold RR of mortality during follow up. Low SDNN appeared to predict mortality benefit from amiodarone (although this effect did not quite achieve significance, $\mathrm{p}=0.075) .{ }^{61}$

In the recent study of La Rovere et al discussed above, ${ }^{58}$ in patients with heart failure, LF power $<13 \mathrm{~ms}^{2}$ and $>83$ ventricular premature beats per hour on Holter monitoring were the two independent predictors of sudden cardiac death during follow up. Sixty two per cent of the study population had neither risk factor, and this group had a very low risk of sudden cardiac death (3\% at three years). In the $38 \%$ of the population with one or two risk factors $23 \%$ of patients died suddenly by three years (fig 6). Importantly, this high predictive accuracy was unaffected by aetiology of heart failure and was still valid in patients taking $\beta$ blockers. ${ }^{58}$ Although this requires confirmation, it suggests that AICDs might be effectively targeted to a high risk subgroup of patients with heart failure.

\section{CONCLUSIONS}

A range of techniques for the assessment of autonomic tone have been described. Impaired baroreflex control of heart period, measured in terms of HRV or BRS, is independently associated with adverse prognosis in patients with heart failure and following acute MI. These techniques have not yet entered routine clinical practice. Their major role in the future may be to define subgroups of patients at high risk of sudden cardiac death, in whom AICD would be justified, and equally, defining a larger subpopulation at relatively low risk.

\section{ACKNOWLEDGEMENTS}

Professor Michael Frenneaux is supported by the British Heart Foundation.

\section{REFERENCES}

1 Rundqvist B, Elam M, Bergmann-Sverrisdottir Y, et al. Increased cardiac adrenergic drive precedes generalized sympathetic activation in human heart failure. Circulation 1997;95:169-75.

2 Schwartz PJ, Vanoli E, Stramba-Badiale M, et al. Autonomic mechanisms and sudden death. New insights from analysis of baroreceptor reflexes in 
conscious dogs with and without a myocardial infarction. Circulation 1988:78:969-79.

3 European Society of Cardiology, NASPE. Heart rate variability: standards of measurement, physiological interpretation and clinical use. Task force of the European Society of Cardiology and the North American Society of Pacing and Electrophysiology. Circulation 1996;93:1043-65

4 Malik M. Geometrical methods for heart rate variability assessment. In: Malik M, Camm AJ, eds. Heart rate variability. Armonk, New York: Futura Publishing, 1995:47-61.

5 Radaelli A, Valle F, Falcone C, et al. Determinants of heart rate variability in heart transplanted subjects during physical exercise. Eur Heart J 1996;17:462-71

6 Lombardi F. Chaos theory, heart rate variability, and arrhythmic mortality. Circulation 2000;101:8-10.

7 Casadei B, Cochrane S, Johnston J, et al. Piffalls in the interpretation of spectral analysis of the heart rate variability during exercise in humans. Acta Physiol Scand 1995;153:125-31.

8 Sleight P, La Rovere MT, Mortara A, et al. Physiology and pathophysiology of heart rate and blood pressure variability in humans: is power spectral analysis largely an index of baroreflex gain? Clin Sci (Lond) 1995;88:103-9.

9 Roach D, Sheldon A, Wilson W, et al. Temporally localized contributions to measures of large-scale heart rate variability. Am J Physiol 1998;274: H1 465-71.

10 Huikuri HV, Makikallio TH, Peng CK, et al. Fractal correlation properties of $R-R$ interval dynamics and mortality in patients with depressed left ventricular function after an acute myocardial infarction. Circulation 2000;101:47-53.

11 Kingwell BA, Cameron JD, Gillies KJ, et al. Arterial compliance may influence baroreflex function in athletes and hypertensives. Am J Physiol 1995:268: H411-8.

12 Smyth HS, Sleight P, Pickering GW. Reflex regulation of arterial pressure during sleep in man. A quantitative method of assessing baroreflex sensitivity. Circ Res 1969;24:109-21.

13 Hogan N, Kardos A, Paterson DJ, et al. Effect of exogenous nitric oxide on baroreflex function in humans. Am J Physiol 1999;277:H221-7.

14 James MA, Panerai RB, Potter JF. Applicability of new techniques in the assessment of arterial baroreflex sensitivity in the elderly: a comparison with established pharmacological methods. Clin Sci (Lond) 1998;94:245-53.

15 Davies LC, Francis D, Jurak P, et al. Reproducibility of methods for assessing baroreflex sensitivity in normal controls and in patients with chronic heart failure. Clin Sci (Lond) 1999;97:515-22.

16 Pagani $M$, Somers V, Furlan R, et al. Changes in autonomic regulation induced by physical training in mild hypertension. Hypertension 1988;12:600-10.

17 Parati G, Saul JP, Di Rienzo M, et al. Spectral analysis of blood pressure and heart rate variability in evaluating cardiovascular regulation. A critical appraisal. Hypertension 1995;25:1276-86.

18 Robbe HW, Mulder $\mathrm{U}$, Ruddel $\mathrm{H}$, et al. Assessment of baroreceptor reflex sensitivity by means of spectral analysis. Hypertension 1987;10:538-43.

19 Pinna GD, Maestri R, Raczak G, et al. Measuring baroreflex sensitivity from the gain function between arterial pressure and heart period. Clin Sci (Lond) 2002;103:81-8.

20 Colucci WS, Ribeiro JP, Rocco MB, et al. Impaired chronotropic response to exercise in patients with congestive heart failure. Role of postsynaptic betaadrenergic desensitization. Circulation 1989;80:314-23.

21 Laver MS, Okin PM, Larson MG, et al. Impaired heart rate response to graded exercise. Prognostic implications of chronotropic incompetence in the Framingham Heart Study. Circulation 1996:93:1520-6.

22 Imai K, Sato H, Hori M, et al. Vagally mediated heart rate recovery after exercise is accelerated in athletes but blunted in patients with chronic heart failure. J Am Coll Cardiol 1994;24:1529-35.

23 Shetler K, Marcus R, Froelicher VF, et al. Heart rate recovery: validation and methodologic issues. J Am Coll Cardiol 2001:38:1980-7.

24 Mrowka R, Persson PB, Theres $\mathrm{H}$, et al. Blunted arterial baroreflex causes "pathological" heart rate turbulence. Am J Physiol Regul Integr Comp Physiol 2000;279:R1171-5.

25 Schmidt G, Malik M, Barthel P, et al. Heart-rate turbulence after ventricular premature beats as a predictor of mortality after acute myocardial infarction. Lancet 1999;353:1390-6.

26 Kleiger RE, Miller JP, Bigger JT Jr, et al. Decreased heart rate variability and its association with increased mortality after acute myocardial infarction. Am J Cardiol 1987;59:256-62

27 Farrell TG, Odemuyiwa O, Bashir Y, et al. Prognostic value of baroreflex sensitivity testing after acute myocardial infarction. Br Heart $J$ 1992;67:129-37.

28 La Rovere MT, Specchia G, Mortara A, et al. Baroreflex sensitivity, clinical correlates, and cardiovascular mortality among patients with a first myocardial infarction. A prospective study. Circulation 1988;78:816-24

29 Bigger JT, Fleiss JL, Rolnitzky LM, et al. The ability of several short-term measures of RR variability to predict mortality after myocardial infarction. Circulation 1993;88:927-34

30 Ghuran A, Reid F, La Rovere MT, et al. Heart rate turbulence-based predictors of fatal and nonfatal cardiac arrest (the autonomic tone and reflexes after myocardial infarction substudy). Am J Cardiol 2002;89:184-90.

31 Mortara A, La Rovere MT, Pinna GD, et al. Arterial baroreflex modulation of heart rate in chronic heart failure: clinical and hemodynamic correlates and prognostic implications. Circulation 1997;96:3450-8

32 Kearney MT, Fox KA, Lee AJ, et al. Predicting death due to progressive heart failure in patients with mild-to-moderate chronic heart failure. $J \mathrm{Am}$ Coll Cardiol 2002;40:1801-8.
33 Nolan J, Batin PD, Andrews R, et al. Prospective study of heart rate variability and mortality in chronic heart failure: results of the United Kingdom heart failure evaluation and assessment of risk trial (UK-heart). Circulation 1998:98:1510-6.

34 La Rovere MT, Pinna GD, Hohnloser SH, et al. Baroreflex sensitivity and heart rate variability in the identification of patients at risk for life-threatening arrhythmias: implications for clinical trials. Circulation 2001;103:2072-7.

35 Wang W, Chen JS, Zucker IH. Carotid sinus baroreceptor sensitivity in experimental heart failure. Circulation 1990;81:1959-66.

36 Ferguson DW, Berg WJ, Sanders JS, et al. Sympathoinhibitory responses to digitalis glycosides in heart failure patients. Direct evidence from sympathetic neural recordings. Circulation 1989:80:65-77.

$37 \mathrm{Krum} \mathrm{H}$, Bigger JT Jr, Goldsmith RL, et al. Effect of long-term digoxin therapy on autonomic function in patients with chronic heart failure. J Am Coll Cardiol 1995;25:289-94.

38 The effect of digoxin on mortality and morbidity in patients with heart failure. The Digitalis Investigation Group. N Engl J Med 1997;336:525-33.

39 Rathore SS, Curtis JP, Wang Y, et al. Association of serum digoxin concentration and outcomes in patients with heart failure. JAMA 2003;289:871-8.

40 Parker GW, Michael LH, Hartley CJ, et al. Central beta-adrenergic mechanisms may modulate ischemic ventricular fibrillation in pigs. Circ Res 1990;66:259-70

41 Lin JL, Chan HL, Du CC, et al. Long-term beta-blocker therapy improves autonomic nervous regulation in advanced congestive heart failure: a longitudinal heart rate variability study. Am Heart $J$ 1999;137:658-65

42 Pousset F, Copie X, Lechat P, et al. Effects of bisoprolol on heart rate variability in heart failure. Am J Cardiol 1996:77:612-7.

43 Sanderson JE, Yeung LY, Chan S, et al. Effect of beta-blockade on baroreceptor and autonomic function in heart failure. Clin Sci (Lond) 1999;96:137-46

44 Lumbers ER, McCloskey DI, Potter EK. Inhibition by angiotensin II of baroreceptor-evoked activity in cardiac vagal efferent nerves in the dog. $J$ Physiol 1979;294:69-80.

45 Potter EK, Reid IA. Intravertebral angiotensin II inhibits cardiac vagal efferent activity in dogs. Neuroendocrinology 1985:40:493-6.

46 Binkley PF, Haas GJ, Starling RC, et al. Sustained augmentation of parasympathetic tone with angiotensin-converting enzyme inhibition in patients with congestive heart failure. J Am Coll Cardiol 1993;21:655-61.

47 Bonaduce $D$, Marciano F, Petretta $M$, et al. Effects of converting enzyme inhibition on heart period variability in patients with acute myocardial infarction. Circulation 1994;90:108-13.

48 Flapan AD, Nolan J, Neilson JM, et al. Effect of captopril on cardiac parasympathetic activity in chronic cardiac failure secondary to coronary artery disease. Am J Cardiol 1992;69:532-5.

49 Vaile JC, Chowdhary S, Osman F, et al. Effects of angiotensin II (ATI) receptor blockade on cardiac vagal control in heart failure. Clin Sci (Lond) 2001:101:559-66.

50 Wang W, McClain JM, Zucker IH. Aldosterone reduces baroreceptor discharge in the dog. Hypertension 1992;19:270-7.

51 Yee KM . Struthers AD. Aldosterone blunts the baroreflex response in man. Clin Sci (Lond) 1998:95:687-92.

52 MacFadyen RJ, Barr CS, Struthers AD. Aldosterone blockade reduces vascular collagen turnover, improves heart rate variability and reduces early morning rise in heart rate in heart failure patients. Cardiovasc Res 1997:35:30-4

53 Katona PG, Lipson D, Dauchot PJ. Opposing central and peripheral effects of atropine on parasympathetic cardiac control. Am J Physiol 1977; 232:H146-51.

54 Casadei B, Conway J, Forfar C, et al. Effect of low doses of scopolamine on RR interval variability, baroreflex sensitivity, and exercise performance in patients with chronic heart failure. Heart 1996;75:274-80.

55 De Ferrari GM, Mantica M, Vanoli E, et al. Scopolamine increases vagal tone and vagal reflexes in patients after myocardial infarction. J Am Coll Cardiol 1993;22:1327-34.

56 La Rovere MT, Mortara A, Pantaleo P, et al. Scopolamine improves autonomic balance in advanced congestive heart failure. Circulation 1994;90:838-43.

57 Pedretti R, Colombo E, Sarzi BS, et al. Influence of transdermal scopolamine on cardiac sympathovagal interaction after acute myocardial infarction. Am J Cardiol 1993;72:384-92.

58 La Rovere MT, Pinna GD, Maestri R, et al. Short-term heart rate variability strongly predicts sudden cardiac death in chronic heart failure patients. Circulation 2003;107:565-70

59 Coletta A, Thackray S, Nikitin N, et al. Clinical trials update: highlights of the scientific sessions of The American College of Cardiology 2002: LIFE, DANAMI 2, MADIT-2, MIRACLE-ICD, OVERTURE, OCTAVE, ENABLE 1 \& 2, CHRISTMAS, AFFIRM, RACE, WIZARD, AZACS, REMATCH, BNP trial and HARDBALL. Eur I Heart Fail 2002;4:381-8.

60 Pinna GD, La Rovere MT, Maestri R, et al. Comparison between invasive and non-invasive measurements of baroreflex sensitivity; implications for studies on risk stratification affer a myocardial infarction. Eur Heart $J$ 2000;21:1522-9

61 Malik M, Camm AJ, Janse MJ, et al. Depressed heart rate variability identifies postinfarction patients who might benefit from prophylactic treatment with amiodarone: a substudy of EMIAT (the european myocardial infarct amiodarone trial). J Am Coll Cardiol 2000;35:1263-75. 